\title{
A Proposal for Revisiting Ćirić and Caristi Type Theorems in Metric Spaces
}

\author{
Belkassem Seddoug, ${ }^{1}$ Soumia Chaira, ${ }^{2}$ and Karim Chaira ${ }^{3}{ }^{3}$ \\ ${ }^{1}$ CRMEF, Rabat-Salé-Kénitra, Avenue Allal El Fassi, Bab Madinat Al Irfane, B.P 6210, 10000 Rabat, Morocco \\ ${ }^{2}$ Laboratory of Mathematics and Applications, Faculty of Sciences and Technologies, University Hassan II Casablanca, \\ Mohammedia, Morocco \\ ${ }^{3}$ Laboratory of Algebra, Analysis and Applications (L3A) Hassan II University of Casablanca, Faculty of Sciences Ben M'Sik, \\ Avenue Cdt Driss El Harti, B.P 7955, Sidi Othmane, Casablanca, Morocco
}

Correspondence should be addressed to Karim Chaira; chaira_karim@yahoo.fr

Received 4 April 2020; Revised 21 May 2020; Accepted 29 May 2020; Published 1 July 2020

Academic Editor: Jean Michel Rakotoson

Copyright $\odot 2020$ Belkassem Seddoug et al. This is an open access article distributed under the Creative Commons Attribution License, which permits unrestricted use, distribution, and reproduction in any medium, provided the original work is properly cited.

In this paper, we revisit the renowned fixed point theorems of Ćirić and Caristi. We propose some new fixed point theorems in a metric space with partial order. To make our results effective, several examples are presented.

\section{Introduction and Preliminary}

This work is motivated by some recent works on the extension of Banach Contraction Principle to metric spaces with a partial order [1]. Caristi's fixed point theorem is maybe one of the most useful extension of Banach Contraction Principle [2-4]. It has been successfully applied in many topics such as differential equations, convex minimization, operator theory, variational inequalities, and control theory. For known Caristi-type fixed point results in the literature, see [5-13]. Recall that this theorem states that any map $T: E \longrightarrow E$ has a fixed point provided that $E$ is complete and there exists a lower semicontinuous map $\varphi: E \longrightarrow[0,+\infty[$ such that $d(x, T x) \leq \varphi(x)-\varphi(T x)$, for every $x \in E$. The proofs given to Caristi's result vary and use different techniques (see $[14,15])$.

Using the combined Ćirić-Caristi condition, we introduce new fixed point theorems under hypotheses of the form

$$
d(T x, T y)
$$

or $d(T x, x) d(T x, T y) \leq$ Dominated Function,

where the "Dominated Function" can be chosen to be

$$
\begin{aligned}
& \qquad \varphi(x)-\varphi(T x)) S(x, y) \\
& \text { or }(\varphi(x)-\varphi(T x)) N(x, y) \\
& \text { or }(\varphi(x)-\varphi(T x)) \max \{1, S(x, y)\},
\end{aligned}
$$

for certain functions $S(x, y)$ and $N(x, y)$. Other corresponding forms under some advanced settings such as "partial order" are also discussed. To the best of our knowledge, we provide all the possible conditions to make the Caristi-type fixed point theorems appropriate and applicable in most situations.

More precisely, the renowned results $[14,16,17]$ for a single-valued map are the following.

Theorem 1 (see Theorem 1 in [16]). Let $(E, d)$ be a complete metric space and $T: E \longrightarrow E$ be a mapping. Suppose that there exists $k \in[0,1$ [ such that

$$
d(T x, T y) \leq k N(x, y)
$$

for all $x, y \in E$, where

$$
N(x, y)=\max \{d(x, y), d(x, T x), d(y, T y), d(y, T x), d(x, T y)\} .
$$

Then, $T$ has a unique fixed point in $E$. 
Definition 1. Let $(E, d,<)$ be a partially ordered complete metric space. We say that $E$ verifies the condition (OSC) if for any decreasing sequence $\left(x_{n}\right)_{n \in \mathbb{N}}$ in $E$ such that $\lim _{n \longrightarrow+\infty} x_{n}$ exists, then there exists $\inf _{n} x_{n}$ and $\inf _{n} x_{n}=\lim _{n \longrightarrow+\infty} x_{n}$.

Theorem 2 (see Theorem 5 in [14]). Let $(E,<)$ be a partially ordered set and suppose that there exists a distance $d$ in $E$ such that $(E, d)$ is a complete metric space satisfying the (OSC) property. Let $T: E \longrightarrow E$ be a monotone increasing mapping. Assume there exists a lower semicontinuous function $\varphi: E \longrightarrow[0,+\infty[$ such that

$$
\{d(x, T x) \leq \varphi(x)-\varphi(T x), \text { whenever } T x<x\} .
$$

Then, $T$ has a fixed point if and only there exists $x_{0} \in E$ such that $T x_{0}<x_{0}$.

In this article, we prove new fixed point theorems of Caristi type and Ćirić type. All optional conditions for dominated functions are presented and discussed.

\section{Main Results}

Theorem 3. Let $(E, d,<)$ be a partially ordered complete metric space satisfying the (OSC) property and $T: E \longrightarrow E$ be a monotonically increasing map such that there exists a function $\varphi: E \longrightarrow[0,+\infty[$ satisfying

$\forall x, y \in E, x<y \Longrightarrow d(x, T x) d(T x, T y) \leq(\varphi(x)-\varphi(T x)) d(x, y)$.

Then, for any $x_{0} \in E$ such that $T x_{0}<x_{0}$, the sequence $\left(x_{n}\right)_{n \in \mathbb{N}}$ defined by $x_{n+1}=T x_{n}$ converges to a fixed point of $T$.

Proof. On the one hand, the case, where there exists $n \in \mathbb{N}$, such that $x_{n}=T x_{n}$, gives $x_{n}$ as a fixed point of $T$.

On the other hand, if $x_{n} \neq T x_{n}$ for every $n \in \mathbb{N}$, then, by induction, the sequence $\left(x_{n}\right)_{n \in \mathbb{N}}$ is monotonically decreasing, and taking $x=x_{n+1}$ and $y=x_{n}$ in (6), one obtains, for every $n \in \mathbb{N}$,

$$
\begin{aligned}
& 0<\frac{\left[d\left(x_{n+1}, x_{n+2}\right)\right]^{2}}{d\left(x_{n}, x_{n+1}\right)} \leq \varphi\left(x_{n+1}\right)-\varphi\left(x_{n+2}\right), \\
& \sum_{k=0}^{n} \frac{\left[d\left(x_{k+1}, x_{k+2}\right)\right]^{2}}{d\left(x_{k}, x_{k+1}\right)} \leq \sum_{k=0}^{n}\left(\varphi\left(x_{k+1}\right)-\varphi\left(x_{k+2}\right)\right) .
\end{aligned}
$$

Since the sequence $\left(\varphi\left(x_{n}\right)\right)_{n \in \mathbb{N}}$ is necessarily positive and monotonically decreasing, the series $\sum_{n \geq 0}\left[\varphi\left(x_{n}\right)-\varphi\left(x_{n+1}\right)\right]$ is convergent and, by the comparison principle, $\sum_{n \geq 0}\left[d\left(x_{n+1}, x_{n+2}\right)\right]^{2} / d\left(x_{n}, x_{n+1}\right)$ is also convergent. Since, for every $k \in \mathbb{N}$, one has

$$
2 d\left(x_{k+1}, x_{k+2}\right) \leq \frac{\left[d\left(x_{k+1}, x_{k+2}\right)\right]^{2}}{d\left(x_{k}, x_{k+1}\right)}+d\left(x_{k}, x_{k+1}\right) .
$$

So, for every $n \in \mathbb{N}$, one obtains the inequality

$$
\sum_{k=1}^{n+1} d\left(x_{k}, x_{k+1}\right) \leq \sum_{k=0}^{n} \frac{\left[d\left(x_{k+1}, x_{k+2}\right)\right]^{2}}{d\left(x_{k}, x_{k+1}\right)}+d\left(x_{0}, x_{1}\right) \text {, }
$$

concluding the convergence of the series $\sum_{n \geq 0} d\left(x_{n}, x_{n+1}\right)$. Hence, $\left(x_{n}\right)_{n \in \mathbb{N}}$ is a Cauchy sequence. Set $x=\lim _{n \longrightarrow \infty} x_{n}$. Since $E$ has the (OSC) property, we obtain $x<x_{n}$ for all $n \in \mathbb{N}$. By (6), one has

$$
d(x, T x) d\left(T x, T x_{n}\right) \leq(\varphi(x)-\varphi(T x)) d\left(x, x_{n}\right),
$$

and so $\lim _{n \longrightarrow \infty} d(x, T x) d\left(T x, T x_{n}\right)=0$. Therefore, $d(x, T x)=0$.

Example 1. Let $E=[0,1]$ be endowed with the usual distance $d(x, y)=|x-y|$ and the order " $<$ " be defined by

$$
\begin{gathered}
\forall(x, y) \in E^{2}, \\
(x<y \Leftrightarrow x \geq y) .
\end{gathered}
$$

Let $T$ and $\varphi$ be two functions defined on $E$ by

$$
\begin{aligned}
& T x= \begin{cases}\frac{x+1}{2}, & \text { if } x \in] 0,1], \\
0, & \text { if } x=0,\end{cases} \\
& \varphi(x)=\frac{1-x}{2}, \quad \text { if } x \in[0,1],
\end{aligned}
$$

where $T$ is monotonically increasing on $E$.

We need only to consider two cases to check the hypothesis of Theorem 3 .

Case 1: let $x, y \in] 0,1]$ with $x<y$. We have

$$
\left(\frac{1-x}{2}\right)\left(\frac{x-y}{2}\right) \leq\left(\frac{1-x}{2}-\frac{1-x}{4}\right)(x-y) .
$$

So,

$$
d(x, T x) d(T x, T y) \leq(\varphi(x)-\varphi(T x)) d(x, y) .
$$

Case 2: $x=0$ and $y \in] 0,1]$. We have

$$
d(0, T 0) d(T 0, T y)=0=(\varphi(0)-\varphi(T 0)) d(0, y)
$$

Remark 1

(i) In Example 1, $T$ does not satisfy the Banach Contraction Principle, for which we take $x=0$ and $y=1 / 3$, and we have $d(T 0, T(1 / 3))>k d(0,1 / 3)$ for all $k \in[0,1[$.

(ii) In Example 1, $T$ does not satisfy the inequality of Caristi, for which we take $x \in] 0,1$ [, and we have $d(x, T x)=(1-x) / 2$ and $\varphi(x)-\varphi(T x)=(1-x) / 4$. So, $d(x, T x)=(1-x) / 2>\varphi(x)-\varphi(T x)$.

Corollary 1. Let $(E, d,<)$ be a partially ordered complete metric space satisfying the (OSC) property and T: E $\longrightarrow E$ be 
a monotonically increasing map such that there exists a function $\varphi: E \longrightarrow[0,+\infty[$ satisfying

$$
\forall x, y \in E, x<y \Longrightarrow d(x, T x) d(T x, T y) \leq\left(\varphi(x)-\varphi\left(T^{p} x\right)\right) d(x, y),
$$

for some $p \in \mathbb{N} \backslash\{0\}$. Then, for any $x_{0} \in E$ such that $T x_{0}<x_{0}$, the sequence $\left(x_{n}\right)_{n \in \mathbb{N}}$, defined by $x_{n+1}=T x_{n}$, converges to $a$ fixed point of $T$.

Proof. If we consider the function $\Phi$ defined on $E$ by $\Phi(x)=\sum_{k=0}^{p-1} \varphi\left(T^{k} x\right)$ for all $x \in E$, we obtain $d(x, T x) d \times$ $(T x, T y) \leq(\Phi(x)-\Phi(T x)) d(x, y)$. By applying Theorem 3 , we get that $T$ admits a fixed point.

Example 2. Let $E=[0,+\infty$ [ endowed with the usual distance $d(x, y)=|x-y|$ and the order " $<$ " defined by

$$
\begin{array}{r}
\forall(x, y) \in E^{2}, \\
(x<y \Longleftrightarrow x \geq y) .
\end{array}
$$

Let $T$ and $\varphi$ be two functions defined on $E$ by

$$
\begin{aligned}
& T x= \begin{cases}\frac{x+1}{2}, & \text { if } x \in] 0,1], \\
x, & \text { if } x \in\{0\} \cap 1,+\infty[,\end{cases} \\
& \varphi(x)= \begin{cases}\frac{2}{3 x}, & \text { if } x \in 0,+\infty[, \\
0, & \text { if } x=0,\end{cases}
\end{aligned}
$$

where $E$ has the condition (OSC) and $T$ is monotonically increasing on $E$.

We need only to consider five cases to check the hypothesis of Corollary 1:

Case 1: $x, y \in] 0,1]$ with $x<y$. We have $T^{2} x=(x+3) / 4$ and

$$
\left(\frac{1-x}{2}\right)\left(\frac{x-y}{2}\right) \leq \frac{2}{3}\left(\frac{1}{x}-\frac{4}{x+3}\right)(x-y)
$$

So,

$d(x, T x) d(T x, T y) \leq\left(\varphi(x)-\varphi\left(T^{2} x\right)\right) d(x, y)$.

Case 2: $y \in] 0,1]$ and $x \in] 1,+\infty[$. We have $d(x, T x)=0$ and $\varphi(x)-\varphi\left(T^{2} x\right)=0$, so

$d(x, T x) d(T x, T y) \leq\left(\varphi(x)-\varphi\left(T^{2} x\right)\right) d(x, y)$.

Case 3: $y=0$ and $x \in] 0,1]$. We have $(1+x)(3+x) \leq 8$ and $T^{2}(x)=(x+3) / 4$, so

$$
\left(\frac{1-x}{2}\right)\left(\frac{x+1}{2}\right) \leq \frac{2}{3}\left(\frac{1}{x}-\frac{4}{x+3}\right) x
$$

Hence,

$d(x, T x) d(T x, T 0) \leq\left(\varphi(x)-\varphi\left(T^{2} x\right)\right) d(x, 0)$.

Case 4: $y=0$ and $x \in] 1,+\infty\left[\right.$. We have $T^{2}(x)=x$, then $d(x, T x)=0=\varphi(x)-\varphi\left(T^{2} x\right)$. So,

$d(x, T x) d(T x, T 0) \leq\left(\varphi(x)-\varphi\left(T^{2} x\right)\right) d(x, 0)$.

Case 5: $x, y \in] 1,+\infty\left[\right.$ with $x<y$. We have $T^{2}(x)=x$, then $d(x, T x)=0=\varphi(x)-\varphi\left(T^{2} x\right)$. So,

$$
d(x, T x) d(T x, T y) \leq\left(\varphi(x)-\varphi\left(T^{2} x\right)\right) d(x, y) .
$$

This shows that inequality (16) is satisfied and all the hypotheses of Corollary 1 are verified. In addition, $T$ has the fixed points 0 and every $x \in[1,+\infty[$.

Remark 2

(i) Example 2 does not satisfy the inequality for $p=1$. If we take the third case $y=0$ and $x=2 / 3$, we have $d(2 / 3, t \operatorname{Tn}(2 / 3)) d(T(2 / 3), T 0)>(\varphi(2 / 3)-\varphi(T(2 /$ 3))) $d(2 / 3, t 0)$.

(ii) Example 2 does not verify the inequality of Theorem 4 in [11]. If we take $y=0$ and $x=2 / 3$, we have $d(2 / 3, t \operatorname{Tn}(2 / 3))>0$ but $d(T(2 / 3), T 0)>(\varphi(2 / 3)-$ $\varphi(T(2 / 3))) d(2 / 3, t 0)$.

(iii) Note that $d(T 0, T(1 / 2))=3 / 4>d(0,1 / 2)=1 / 2$, so the mapping $T$ is not nonexpansive.

Definition 2. Let $(E, d,<)$ be a partially ordered complete metric space and $T: E \longrightarrow E$ be a mapping. We say that $T$ satisfies the condition $(w c)$ if, for any monotone decreasing sequence $\left(x_{n}\right)_{n \in \mathbb{N}}$ in $E$ such that there exists $x=\lim _{n \longrightarrow+\infty} x_{n}$ and $x<x_{n}$ for all $n \in \mathbb{N}$, we have $\lim _{n \longrightarrow+\infty} d\left(x, T x_{n}\right)=0$.

Theorem 4. Let $(E, d,<)$ be a partially ordered complete metric space and a monotonically increasing map $T: E \longrightarrow E$ such that there exists a function $\varphi: E \longrightarrow[0,+\infty[$ satisfying $\forall x, y \in E, x<y \Longrightarrow d(x, T x) d(T x, T y) \leq(\varphi(x)-\varphi(T x)) S(x, y)$,

where

$$
S(x, y)=d(x, y)+d(x, T x)+d(y, T y) .
$$

Assume that there exists a point $x_{0} \in E$ satisfying $T x_{0}<x_{0}$. If we further add one of the following hypotheses,

(C1) $T$ is continuous

(C2) The map $x \mapsto d(x, T x)$ is lower semicontinuous

(C3) $E$ has the condition (OSC), $T$ has the condition $(w c)$, and $\varphi$ is lower semicontinuous then $T$ has at least one fixed point. 
Proof. We define the sequence $\left(x_{n}\right)_{n \geq 0}$ by $x_{n+1}=T x_{n}$ for each $n \in \mathbb{N}$. On the one hand, the case where there exists $n \in \mathbb{N}$, such that $x_{n}=T x_{n}$, gives $x_{n}$ as a fixed point of $T$. On the other hand, if $x_{n} \neq T x_{n}$ for every $n \in \mathbb{N}$, then, by induction, the sequence $\left(x_{n}\right)_{n \in \mathbb{N}}$ is monotonically decreasing, and taking $x=x_{n+1}$ and $y=x_{n}$ in (3), one obtains, for every $n \in \mathbb{N}$

$$
0<\frac{\left[d\left(x_{n+1}, x_{n+2}\right)\right]^{2}}{S\left(x_{n}, x_{n+1}\right)} \leq \varphi\left(x_{n+1}\right)-\varphi\left(x_{n+2}\right),
$$

where $S\left(x_{n}, x_{n+1}\right)=2 d\left(x_{n}, x_{n+1}\right)+d\left(x_{n+1}, x_{n+2}\right)$. So, the sequence $\left(\varphi\left(x_{n}\right)\right)_{n \in \mathbb{N}}$ is necessarily positive and monotonically decreasing, and therefore the series $\sum_{n \geq 0}\left[\varphi\left(x_{n}\right)-\varphi\left(x_{n+1}\right)\right] \quad$ is convergent. Thus, $\sum_{n \geq 0}\left[d\left(x_{n+1}, x_{n+2}\right)\right]^{2} / S\left(x_{n}, x_{n+1}\right)$ is also convergent. For every $k \in \mathbb{N}$, one has

$$
\begin{aligned}
4 d\left(x_{k+1}, x_{k+2}\right) \leq & \frac{4\left[d\left(x_{k+1}, x_{k+2}\right)\right]^{2}}{2 d\left(x_{k}, x_{k+1}\right)+d\left(x_{k+1}, x_{k+2}\right)} \\
& +2 d\left(x_{k}, x_{k+1}\right)+d\left(x_{k+1}, x_{k+2}\right) .
\end{aligned}
$$

Therefore, for every $n \in \mathbb{N}$, one obtains the inequality

$$
\sum_{k=1}^{n+1} d\left(x_{k}, x_{k+1}\right) \leq 4 \sum_{k=0}^{n} \frac{\left[d\left(x_{k+1}, x_{k+2}\right)\right]^{2}}{2 d\left(x_{k}, x_{k+1}\right)+d\left(x_{k+1}, x_{k+2}\right)}+2 d\left(x_{0}, x_{1}\right) \text {, }
$$

obtaining the convergence of the series $\sum_{n \geq 0} d\left(x_{n}, x_{n+1}\right)$. Hence, $\left(x_{n}\right)_{n \in \mathbb{N}}$ is a Cauchy sequence. Set $x^{*}=\lim _{n \rightarrow \infty} x_{n}$.

(C1) and (C2) The case where $T$ is continuous is obvious. If the function $x \mapsto d(x, T x)$ is lower semicontinuous, we obtain

$$
d\left(x^{*}, T x^{*}\right) \leq \liminf _{n \longrightarrow+\infty} d\left(x_{n}, T x_{n}\right)=\lim _{n \longrightarrow+\infty} d\left(x_{n}, x_{n+1}\right)=0 .
$$

Thus, $d\left(x^{*}, T x^{*}\right)=0$, i.e., $T x^{*}=x^{*}$.

(C3) Suppose $E$ has the condition (OSC), so there exists $\inf _{n \in \mathbb{N}} x_{n}$ and is equal to $x^{*}$. We have $x^{*}<x_{n}$ for all $n \in \mathbb{N}$. Since $T$ is strictly increasing, $T x^{*}<T x_{n}=x_{n+1}$, thus $T x^{*}<\inf _{n \in \mathbb{N}} x_{n}=x^{*}$.

In what follows, we suppose that $T x^{*} \neq x^{*}$ (because otherwise $T$ admits $x^{*}$ as a fixed point).

We put $E_{o}=\left\{x \in E, T x<x\right.$ and $\left.x<x^{*}\right\}$. The set $E_{o}$ is nonempty because $x^{*} \in E_{o}$. Let $x \in E_{o}$, so $x<x^{*}$. Since $T$ is monotonically increasing, $T x<T x^{*}<x^{*}$, thus $T\left(E_{o}\right) \subset E_{o}$.

Taking $x=x^{*}$ and $y=x_{n}$ in inequality (26), we have

$$
d\left(x^{*}, T x^{*}\right) d\left(T x^{*}, T x_{n}\right) \leq\left(\varphi\left(x^{*}\right)-\varphi\left(T x^{*}\right)\right) S\left(x^{*}, x_{n}\right) \text {. }
$$

Letting $n$ tend to $+\infty$, we obtain

$$
\left(d\left(x^{*}, T x^{*}\right)\right)^{2} \leq\left(\varphi\left(x^{*}\right)-\varphi\left(T x^{*}\right)\right) d\left(x^{*}, T x^{*}\right) .
$$

By (33), we obtain

$$
d\left(x^{*}, T x^{*}\right) \leq \varphi\left(x^{*}\right)-\varphi\left(T x^{*}\right) .
$$

We define the partial order “ $\prec$ ” on $E_{o}$ by

$$
x \prec y \Leftrightarrow[x<y \text { and } d(x, y) \leq \varphi(y)-\varphi(x)],
$$

where " $\leq$ " is the usual order on $\mathbb{R}$. It is interesting to see that $\varphi$ is monotonically increasing from $E_{o}$ to $[0,+\infty[$.

Assume that $\left\{F_{\beta}\right\}_{\beta \in \Gamma}$ is a set of totally ordered subset of $E_{o}$ such that, for any $\beta_{1}, \beta_{2} \in \Gamma, F_{\beta_{1}} \subset F_{\beta_{2}}$, or $F_{\beta_{2}} \subset F_{\beta_{1}}$, where $\Gamma$ is the index set. Let $G=\cup_{\beta \in \Gamma} F_{\beta}$. For any $x, y \in G$ there exists $\beta_{x}, \beta_{y} \in \Gamma$ such that $x \in F_{\beta_{x}}$ and $y \in F_{\beta_{y}}$. We may assume that $F_{\beta_{x}} \subset F_{\beta_{y}}$, so $x, y \in F_{\beta_{y}}$, that is, $x$ and $y$ are comparable. Hence, $G$ is totally ordered subset of $E_{o}$, and by Zorn's lemma, $E_{o}$ has a maximal totally ordered subset.

Let $F$ be a maximal totally ordered subset of $E_{o}$. We consider $\varphi_{0}=\inf _{x \in F} \varphi(x)$ and a sequence $\left(y_{p}\right)_{p \in \mathbb{N}} \subset F$ such that $\left(\varphi\left(y_{p}\right)\right)_{p \in \mathbb{N}}$ is decreasing and convergent to $\varphi_{0}$. By monotony of $\varphi$ on $E_{o}$, the sequence $\left(y_{p}\right)_{p \in \mathbb{N}}$ is also decreasing in $E_{o}$. Therefore, for every integers $p \leq q$, we have $d\left(y_{p}, y_{q}\right) \leq \varphi\left(y_{p}\right)-\varphi\left(y_{q}\right)$, which implies that $\left(y_{p}\right)_{p \in \mathbb{N}}$ is a Cauchy's sequence and there exists a unique $y^{*} \in E$ such that $\lim _{p \longrightarrow \infty} y_{p}=y^{*}$. So, $\inf _{p} y_{p}=\lim _{p \longrightarrow+\infty} y_{p}=y^{*}$, that is, $y^{*}<y_{p}$ for all $p \in \mathbb{N}$. Moreover, by the lower semicontinuity of $\varphi$, we obtain $\varphi\left(y^{*}\right) \leq \liminf \operatorname{in}_{p \longrightarrow+\infty} \varphi\left(y_{p}\right)=\varphi_{0}$.

Next, we show that $y^{*} \prec z$ for all $z \in F$. For that we distinguish two cases:

Case 1: there exists $x \in F$ satisfying $\varphi(x)=\varphi_{0}$, so we have $x \prec z$, for any $z \in F$. It follows that $x<y_{p}$ and $d\left(x, y_{p}\right) \leq \varphi\left(y_{p}\right)-\varphi_{0}$ for all $p \in \mathbb{N}$. Letting $p$ tend to infinity, we have $d\left(x, y^{*}\right)=0$, which means that $y^{*}=$ $x<z$ for all $z \in F$.

Case 2: let $z \in F, \varphi(z) \neq \varphi_{0} \leq \varphi(z)$, so there exists $N>0$ such that $y_{p} \prec z$ whenever $p \geq N$. Hence, $y^{*}<y_{p}<z$ and $d\left(z, y_{p}\right) \leq \varphi(z)-\varphi\left(y_{p}\right)$ for all $p \geq N$. Letting $p$ tends to infinity, we obtain $d\left(z, y^{*}\right) \leq \varphi(z)-\varphi_{0} \leq \varphi(z)-\varphi\left(y^{*}\right)$, and so $y^{*} \prec z$.

On the basis of the above discussion, we can claim that $y^{*} \prec z$ for all $z \in F$. Hence, $y^{*}$ is lower bound of $F$ in $E_{o}$ with respect to the order " $\prec$." (26),

We have $y^{*}<y_{p}$ for every $p \in \mathbb{N}$. Then, by inequality

$$
\begin{aligned}
d\left(y^{*}, T y^{*}\right) d\left(T y^{*}, T y_{p}\right) & \leq\left(\varphi\left(y^{*}\right)-\varphi\left(T y^{*}\right)\right)\left(d\left(y^{*}, y_{p}\right)\right. \\
& \left.+d\left(y^{*}, T y^{*}\right)+d\left(y_{p}, T y_{p}\right)\right)
\end{aligned}
$$

and so

$$
\begin{array}{r}
d\left(y^{*}, T y^{*}\right)\left(d\left(y^{*}, T y^{*}\right)-d\left(y^{*}, T y_{p}\right)\right) \leq\left(\varphi\left(y^{*}\right)-\varphi\left(T y^{*}\right)\right) \\
\left(2 d\left(y^{*}, y_{p}\right)+d\left(y^{*}, T y^{*}\right)+d\left(y^{*}, T y_{p}\right)\right) .
\end{array}
$$

Since $T$ has the condition $(w c)$, and when $p$ tends to infinity, we obtain

$$
\left[d\left(y^{*}, T y^{*}\right)\right]^{2} \leq\left(\varphi\left(y^{*}\right)-\varphi\left(T y^{*}\right)\right) d\left(y^{*}, T y^{*}\right) .
$$

We claim that $T y^{*}=y^{*}$. Indeed, if $T y^{*} \neq y^{*}$, one has

$$
d\left(y^{*}, T y^{*}\right) \leq \varphi\left(y^{*}\right)-\varphi\left(T y^{*}\right) \text {. }
$$


Since $T$ is monotonically increasing, we have $T y^{*}<T x<x$ for all $x \in F$. Particularly, $T y^{*}<y_{p}$ for all $p \in \mathbb{N}$, which implies that $T y^{*}<\inf _{p} y_{p}=y^{*}$. Thus, $y^{*} \in E_{o}$ and, by the fact that $T\left(E_{o}\right) \subset E_{o}$, we get $T y^{*} \in E_{o}$ and $T y^{*} \prec y^{*} \prec z$ for all $z \in F$. Since $T y^{*} \neq y^{*}$, we get that $T y^{*} \in F$ and $\left\{T y^{*}, y^{*}\right\} \cup F$ is a totally ordered subset of $E_{o}$. This contradicts the maximality of $F$ and finishes the proof.

Example 3. Let $E=\left\{1 / n: t n n \in q \mathbb{N}^{*}\right\} \cup\{0\}$ endowed with the usual distance $d(x, y)=|x-y|$ and the usual order " $<$." We put, for all $n \in \mathbb{N} \backslash\{0\}, x_{n}=1 / n$ and $x_{0}=0$. Let $T$ and $\varphi$ be the two functions defined on $E$ by

$$
\begin{gathered}
T x_{n}= \begin{cases}x_{n+1}, & \text { if } n \in \mathbb{N} \backslash\{0\}, \\
0, & \text { if } n=0,\end{cases} \\
\varphi\left(x_{n}\right)= \begin{cases}2 x_{n}, & \text { if } n \in \mathbb{N} \backslash\{1\}, \\
x_{1}, & \text { if } n=1,\end{cases}
\end{gathered}
$$

where $(E, d)$ is a complete metric space and verifies the condition (OSC). $T$ is monotone increasing on $E$ and has the condition ( $w c)$ (actually, $T$ is continuous), $\varphi$ is lower semicontinuous on $E$, and $T x_{1} \leq x_{1}$.

We need only to consider two cases to check the hypothesis of Theorem 4:

Case 1: let $n, m \in \mathbb{N} \backslash\{0\}$ such that $m<n$ and $n \neq m$. We have $x_{n} \leq x_{m}$ and $(1 /(n+1))+(3 /(m+1)) \leq 4 / m$, i.e., $x_{n+1}+3 x_{m+1} \leq 4 x_{m}$. So,

$$
\begin{aligned}
x_{m+1}-x_{n+1} & \leq 2\left[\left(x_{m}-x_{n}\right)+\left(x_{n}-x_{n+1}\right)+\left(x_{m}-x_{m+1}\right)\right], \\
\left(x_{n}-x_{n+1}\right)\left(x_{m+1}-x_{n+1}\right) & \leq\left(\varphi\left(x_{n}\right)-\varphi\left(x_{n+1}\right)\right)\left[\left(x_{m}-x_{n}\right)+\left(x_{n}-x_{n+1}\right)+\left(x_{m}-x_{m+1}\right)\right] .
\end{aligned}
$$

Thus,

$$
d\left(x_{n}, T x_{n}\right) d\left(T x_{m}, T x_{n}\right) \leq\left(\varphi\left(x_{n}\right)-\varphi\left(T x_{n}\right)\right) S\left(x_{m}, x_{n}\right) .
$$

Case 2: $x=x_{0}$ and $y=x_{n}, n \in \mathbb{N} \backslash\{0\}$. We have $d\left(x_{0}, T x_{0}\right)=0=\varphi\left(x_{0}\right)-\varphi\left(T x_{0}\right)$. So,

$$
d\left(x_{0}, T x_{0}\right) d\left(T x_{0}, T x_{n}\right)=\left(\varphi\left(x_{0}\right)-\varphi\left(T x_{0}\right)\right) S\left(x_{0}, x_{n}\right) .
$$

This shows that inequality (26) is satisfied and all the hypotheses hold (including condition (C3)). In addition, $T$ has the fixed point 0 .

Note that $d\left(x_{1}, T x_{1}\right)>\varphi\left(x_{1}\right)-\varphi\left(T x_{1}\right)$, then $T$ does not satisfy the inequality of Caristi.

Corollary 2. Let $(E, d,<)$ be a partially ordered complete metric space and $T: E \longrightarrow E$ be a monotone increasing map such that there exists a function $\varphi: E \longrightarrow[0,+\infty[$ satisfying

$\forall x, y \in E, x<y \Longrightarrow d(x, T x) d(T x, T y) \leq(\varphi(x)-\varphi(T x)) N(x, y)$,

where

$N(x, y)=\max \{d(x, y), d(x, T x), d(x, T y), d(T x, y), d(T y, y)\}$.
Assume that there exists a point $x_{0} \in E$ satisfying $T x_{0}<x_{0}$. If we further add one of the hypotheses (C1), (C2), or (C3) of Theorem 4, then $T$ has at least one fixed point.

Corollary 3. Let $(E, d,<)$ be a partially ordered complete metric space and let $T: E \longrightarrow E$ be a monotone increasing map such that there exists a function $\varphi: E \longrightarrow[0,+\infty[$ satisfying

$\forall x, y \in E, x<y \Longrightarrow d(x, T x) d(T x, T y) \leq\left(\varphi(x)-\varphi\left(T^{p} x\right)\right) S(x, y)$,

for some $p \in \mathbb{N}$, where

$$
S(x, y)=d(x, y)+d(x, T x)+d(y, T y) .
$$

Assume that there exists a point $x_{0} \in E$ satisfying $T x_{0}<x_{0}$. If we further add one of the hypotheses (C1), (C2), or (C3) of Theorem 4, replacing the lower semicontinuity of $\varphi$ in (C3) with the lower semicontinuity of $\varphi^{\circ} T^{i}$, for each $i \in\{1, \ldots, p-1\}$, then $T$ has at least one fixed point.

Proof. Let $(x, y) \in E^{2}$ such that $x<y$. By inequality (46), we have

$$
d(x, T x) d(T x, T y) \leq\left(\varphi(x)-\varphi\left(T^{p} x\right)\right) S(x, y) .
$$

If we consider the function $\Phi$ define on $E$ by $\Phi(x)=$ $\sum_{k=0}^{p-1} \varphi\left(T^{k} x\right)$ for all $x \in E$, we obtain $d(x, T x) d(T x, T y)$ $\leq(\Phi(x)-\Phi(T x)) d(x, y)$, and by hypothesis (C3), the function $\Phi: E \longrightarrow[0,+\infty[$ is lower semicontinuous. Applying Theorem 4, we deduce that $T$ admits a fixed point. 
Our goal in the rest of this article is to generalize the recent Theorem 4 in [11] and to give a short proof of it.

Theorem 5. Let $(E, d)$ be a complete metric space and $T: E \longrightarrow E$ a map such that there exists a function $\varphi: E \longrightarrow[0,+\infty[$ satisfying

$$
d(x, T x)>0 \Longrightarrow d(T x, T y) \leq(\varphi(x)-\varphi(T x)) \max \{1, S(x, y)\},
$$

where

$$
S(x, y)=d(x, y)+d(x, T x)+d(y, T y) .
$$

Then, $T$ has a fixed point.

Proof. Let $x_{0} \in E$ and define the sequence $\left(x_{n}\right)_{n \in \mathbb{N}}$ by $x_{n+1}=T x_{n}$. On the one hand, the case where there exists $n \in \mathbb{N}$, such that $x_{n}=T x_{n}$, gives $x_{n}$ as a fixed point of $T$. On the other hand, if $x_{n} \neq T x_{n}$ for every $n \in \mathbb{N}$, then, taking $x=$ $x_{n}$ and $y=x_{n+1}$ in (49), one obtains, for every $n \in \mathbb{N}$,

$$
0<\frac{d\left(x_{n+1}, x_{n+2}\right)}{\max \left\{1, S\left(x_{n}, x_{n+1}\right)\right\}} \leq \varphi\left(x_{n}\right)-\varphi\left(x_{n+1}\right)
$$

where $S\left(x_{n}, x_{n+1}\right)=2 d\left(x_{n}, x_{n+1}\right)+d\left(x_{n+1}, x_{n+2}\right)$, which shows that the sequence $\left(\varphi\left(x_{n}\right)\right)_{n \in \mathbb{N}}$ is necessarily positive and monotonically decreasing. Therefore, the series $\sum_{n \geq 0}\left[\varphi\left(x_{n}\right)-\varphi\left(x_{n+1}\right)\right]$ is convergent, and by the comparison principle, $\quad \sum_{n \geq 0} d\left(x_{n+1}, x_{n+2}\right) / \max \left\{1, S\left(x_{n}, x_{n+1}\right)\right\}$ is also convergent and $\lim _{n \longrightarrow+\infty} d\left(x_{n+1}, x_{n+2}\right) / \max \left\{1, S\left(x_{n}, x_{n+1}\right)\right\}=$ 0 .

Let $k \in] 0,1 / 3]$, there exists $N \in \mathbb{N}$ such that, for all $n \geq N$,

$$
\begin{aligned}
d\left(x_{n+1}, x_{n+2}\right) & \leq k \max \left\{1, S\left(x_{n}, x_{n+1}\right)\right\} \\
& \leq k \max \left\{1,2 d\left(x_{n}, x_{n+1}\right)+d\left(x_{n+1}, x_{n+2}\right)\right\} .
\end{aligned}
$$

So,

$$
\begin{aligned}
d\left(x_{n+1}, x_{n+2}\right) & \leq \frac{2 k}{1-k} \max \left\{1, d\left(x_{n}, x_{n+1}\right)\right\} \\
& \leq \max \left\{1, d\left(x_{n}, x_{n+1}\right)\right\}
\end{aligned}
$$

because $k \in] 0,1 / 3]$. The sequence $\left(d\left(x_{n}, x_{n+1}\right)\right)_{n}$ is then positive and bounded above by

$$
M=\max \left\{1, d\left(x_{0}, x_{1}\right), \ldots, d\left(x_{N}, x_{N+1}\right)\right\} .
$$

Next, we prove that $\left(x_{n}\right)_{n \geq 0}$ is a Cauchy sequence. For this, we present two methods.

Method 1. Since $\left(d\left(x_{n}, x_{n+1}\right)\right)_{n \geq 0}$ is bounded, we get $\lim \sup _{n \longrightarrow+\infty} d\left(x_{n}, x_{n+1}\right)<\infty$. We have

$$
\limsup _{n \longrightarrow+\infty} d\left(x_{n+1}, x_{n+2}\right) \leq \lim _{n \longrightarrow+\infty}\left(\varphi\left(x_{n}\right)-\varphi\left(x_{n+1}\right)\right) \max \left\{1, \limsup _{n \longrightarrow+\infty} S\left(x_{n}, x_{n+1}\right)\right\}=0
$$

Thus, $\lim _{n \longrightarrow+\infty} d\left(x_{n}, x_{n+1}\right)=0$.

Suppose that $\left(x_{n}\right)_{n \geq 0}$ is not a Cauchy sequence. Then, there exists $\varepsilon>0$ for which we can find subsequences $\left(x_{m(k)}\right)_{k \geq 0}$ and $\left(x_{n(k)}\right)_{k \geq 0}$ with $n(k)>m(k)>k$, and $n(k)$ is the smallest integer such that

$$
\begin{array}{r}
d\left(x_{n(k)}, x_{m(k)}\right) \geq \varepsilon, \\
d\left(x_{m(k)}, x_{n(k)-1}\right)<\varepsilon .
\end{array}
$$

$$
\begin{array}{r}
\varepsilon \leq d\left(x_{m(k)}, x_{n(k)}\right) \leq d\left(x_{m(k)}, x_{n(k)-1}\right)+d\left(x_{n(k)-1}, x_{n(k)}\right) \\
\leq \varepsilon+d\left(x_{n(k)-1}, x_{n(k)}\right) .
\end{array}
$$
obtain

Taking $k \longrightarrow \infty$ and using $\lim _{n \longrightarrow+\infty} d\left(x_{n}, x_{n+1}\right)=0$, we

$$
\lim _{k \longrightarrow+\infty} d\left(x_{m(k)}, x_{n(k)}\right)=\varepsilon
$$

Similarly, we have

So,

$$
\begin{aligned}
& d\left(x_{n(k)}, x_{m(k)}\right) \leq d\left(x_{n(k)}, x_{n(k)-1}\right)+d\left(x_{n(k)-1}, x_{m(k)-1}\right)+d\left(x_{m(k)-1}, x_{m(k)}\right) \\
& d\left(x_{n(k)-1}, x_{m(k)-1}\right) \leq d\left(x_{n(k)-1}, x_{n(k)}\right)+d\left(x_{n(k)}, x_{m(k)}\right)+d\left(x_{m(k)}, x_{m(k)-1}\right) .
\end{aligned}
$$


Letting $k \longrightarrow \infty$ in the above two inequalities and using (56), we obtain

$$
\lim _{k \longrightarrow+\infty} d\left(x_{m(k)-1}, x_{n(k)-1}\right)=\varepsilon .
$$
obtains

Now, taking $y=x_{m(k)-1}$ and $x=x_{n(k)-1}$ in (49), one

$$
\begin{aligned}
d\left(T x_{n(k)-1}, T x_{m(k)-1}\right) & \leq\left(\varphi\left(x_{n(k)-1}\right)-\varphi\left(T x_{n(k)-1}\right)\right) \max \left\{1, S\left(x_{n(k)-1}, x_{m(k)-1}\right)\right\} \\
d\left(x_{n(k)}, x_{m(k)}\right) & \leq\left(\varphi\left(x_{n(k)-1}\right)-\varphi\left(x_{n(k)}\right)\right) \times \max \left\{1, d\left(x_{n(k)-1}, x_{m(k)-1}\right)+d\left(x_{n(k)-1}, x_{n(k)}\right)+d\left(x_{m(k)-1}, x_{m(k)}\right)\right\} .
\end{aligned}
$$

Letting $k \longrightarrow \infty$, we obtain

$0<\varepsilon \leq \lim _{k \longrightarrow+\infty}\left[\varphi\left(x_{n(k)-1}\right)-\varphi\left(x_{n(k)}\right)\right] \max \{1, \varepsilon\}=0$,

which is a contradiction. This shows that $\left(x_{n}\right)_{n \geq 0}$ is a Cauchy sequence.

Method 2. We have $\max \left\{1, S\left(x_{n}, x_{n+1}\right)\right\} \leq M^{\prime}=\max \{1,3 M\}$ for all $n \in \mathbb{N}$. So, for all $n \in \mathbb{N}$,

$$
\begin{aligned}
d\left(x_{n+1}, x_{n+2}\right) & =\frac{d\left(x_{n+1}, x_{n+2}\right)}{\max \left\{1, S\left(x_{n}, x_{n+1}\right)\right\}} \times \max \left\{1, S\left(x_{n}, x_{n+1}\right)\right\} \\
& \leq M^{\prime} \frac{d\left(x_{n+1}, x_{n+2}\right)}{\max \left\{1, S\left(x_{n}, x_{n+1}\right)\right\}} .
\end{aligned}
$$

Since $\sum_{n \geq 0} d\left(x_{n+1}, x_{n+2}\right) / \max \left\{1, S\left(x_{n}, x_{n+1}\right)\right\}$ is convergent, the series $\sum_{n \geq 0} d\left(x_{n+1}, x_{n+2}\right)$ is also convergent. Therefore, $\left(d\left(x_{n}, x_{n+1}\right)\right)_{n}$ is a Cauchy sequence. Hence, the sequence $\left(x_{n}\right)_{n \geq 0}$ is convergent. Let $x^{*}=\lim _{n \longrightarrow \infty} x_{n}$. Taking $x=x_{n}$ and $y=x^{*}$ in (49), one obtains, for every $n \in \mathbb{N}$,

$$
d\left(x_{n+1}, T x^{*}\right) \leq\left(\varphi\left(x_{n}\right)-\varphi\left(x_{n+1}\right)\right) \max \left\{1, S\left(x^{*}, x_{n}\right)\right\} .
$$

Letting $n \longrightarrow+\infty$, we have $d\left(x^{*}, T x^{*}\right)=0$.

Example 4. Let $E=[0,1] \cup[(1+\sqrt{2}) / 2,+\infty$ [ with the usual distance $d(x, y)=|x-y|$. Let $T$ and $\varphi$ be the functions defined on $E$ by

$$
\begin{aligned}
& T x= \begin{cases}1, & \text { if } x \in[0,1], \\
\frac{1}{x+1}, & \text { if } x \in\left[\frac{1+\sqrt{2}}{2},+\infty[,\right.\end{cases} \\
& \varphi(x)= \begin{cases}\frac{1}{2}, & \text { if } x \in[0,1[, \\
3, & \text { if } x \in\left[\frac{1+\sqrt{2}}{2},+\infty[,\right. \\
0, & \text { if } x=1 .\end{cases}
\end{aligned}
$$

We need only to consider five cases to check the hypothesis of Theorem 5 .

Case $1: x, y \in[(1+\sqrt{2}) / 2,+\infty[$.

$$
\begin{aligned}
d(T x, T y) & =\frac{|x-y|}{(1+x)(1+y)} \\
& \leq \frac{5}{2}=\varphi(x)-\varphi(T x) \\
& \leq(\varphi(x)-\varphi(T x)) \max \{1, S(x, y)\}, \\
d(T x, T y) & \leq(\varphi(y)-\varphi(T y)) \max \{1, S(x, y)\} .
\end{aligned}
$$

Case 2: $x, y \in[0,1[$.

$$
\begin{aligned}
d(T x, T y) & =0 \\
& \leq \frac{1}{2}=(\varphi x)-\varphi(T x) \\
& \leq(\varphi(x)-\varphi(T x)) \max \{1, S(x, y)\}, \\
d(T x, T y) & \leq(\varphi(y)-\varphi(T y)) \max \{1, S(x, y)\} .
\end{aligned}
$$

Case 3: $x \in[0,1[$ and $y \in[(1+\sqrt{2}) / 2,+\infty[$. We have $y /(1+y) \leq(1 / 2)(y-(y /(1+y)))$ because $y \geq(1+$ $\sqrt{2}) / 2$. So,

$$
\begin{aligned}
d(T x, T y) & \leq(\varphi(x)-\varphi(T x)) d(y, T y) \\
& \leq \max \{1, S(x, y)\}, \\
d(T x, T y) & \leq \frac{5}{2}=\varphi(y)-\varphi(T y) \\
& \leq(\varphi(y)-\varphi(T y)) \max \{1, S(x, y)\} .
\end{aligned}
$$

Case 4: $x=1$ and $y \in[(1+\sqrt{2}) / 2,+\infty[$. We have $d(1, T 1)=0$ and 


$$
\begin{aligned}
d(T 1, T y) & =\frac{y}{1+y} \\
& \leq \frac{5}{2}=\varphi(y)-\varphi(T y) \\
& \leq(\varphi(y)-\varphi(T y)) \max \{1, S(x, y)\}
\end{aligned}
$$

Case 5: $x=1$ and $y \in[0,1[$. We have $d(1, T 1)=0$ and

$$
\begin{aligned}
d(T 1, T y) & =0 \\
& \leq \frac{1}{2}=\varphi(y)-\varphi(T y) \\
& \leq(\varphi(y)-\varphi(T y)) \max \{1, S(x, y)\} .
\end{aligned}
$$

This shows that inequality (49) is satisfied and all the hypotheses are verified. In addition, $T$ has the fixed point 1 .

We notice that, in the third case, $x \in[0,1[$ and $y \in[(1+\sqrt{2}) / 2,+\infty[$, and we have $d(T x, T y)>$ $\varphi(x)-\varphi(T x)$.

Remark 3. If we take Example 1 of [11], $T$ satisfies condition (49) of our theorem and admits a fixed point. However, $T$ does not satisfy the Banach Contraction Principle nor the Ćirić contraction.

Theorem 4 in [11] treats the case, where $\max \{1, S(x, y)\}$ in (49), and is replaced by

$$
N(x, y)=\max \{d(x, y), d(x, T x), d(x, T y), d(T x, y), d(T y, y)\} .
$$

By triangular inequality, we obtain

$$
N(x, y) \leq \max \{1, S(x, y)\}
$$

which gives rise to the following corollary.

Corollary 4. Let $(E, d)$ be a complete metric space and $T: E \longrightarrow E$ a map such that there exists a function $\varphi: E \longrightarrow[0,+\infty[$ satisfying

$$
d(x, T x)>0 \Longrightarrow d(T x, T y) \leq(\varphi(x)-\varphi(T x)) N(x, y),
$$

where

$$
N(x, y)=\max \{d(x, y), d(x, T x), d(x, T y), d(T x, y), d(T y, y)\} .
$$

Then, $T$ has a fixed point.

Corollary 5. Let $(E, d)$ be a complete metric space and $T: E \longrightarrow E$ a map such that there exists a function $\varphi: E \longrightarrow\left[0,+\infty\left[\right.\right.$ satisfying, for all $(x, y) \in E^{2}$,

$$
d(x, T x)>0 \Longrightarrow d(T x, T y) \leq(\varphi(x)-\varphi(T x))(d(x, y))^{\alpha},
$$

for some real $\alpha \in[0,1]$. Then, $T$ has a fixed point.

Proof. If assumption (75) holds for some real $\alpha \in[0,1]$ then (49) holds, since for every $(x, y) \in E^{2}$, one has

$$
(d(x, y))^{\alpha} \leq \max \{1, d(x, y)\} \leq \max \{1, S(x, y)\},
$$

which allows to conclude that $T$ admits a fixed point.

\section{Data Availability}

No data were used to support this study.

\section{Conflicts of Interest}

The authors declare that they have no conflicts of interest.

\section{References}

[1] J. J. Nieto and R. Rodríguez-López, "Contractive mapping theorems in partially ordered sets and applications to ordinary differential equations," Order, vol. 22, no. 3, pp. 223-239, 2005.

[2] A. Brondsted, "Shorter notes: fixed points and partial orders," Proceedings of the American Mathematical Society, vol. 60, no. 1, pp. 365-366, 1976.

[3] A. Brondsted, "Common fixed points and partial orders," Proceedings of the American Mathematical Society, vol. 77, no. 3, pp. 365-368, 1979.

[4] J. Caristi, "Fixed point theorems for mappings satisfying inwardness conditions," Transactions of the American Mathematical Society, vol. 215, p. 241, 1976.

[5] Ö. Acar and I. Altun, "Some generalizations of Caristi type fixed point theorem on partial metric spaces," Filomat, vol. 26, no. 4, pp. 833-837, 2012.

[6] H. Aydi and D. Zhang, "Refinements of Caristis fixed point theorem," Journal of Nonlinear Sciences and Applications, vol. 9, no. 6, pp. 4084-4097, 2016.

[7] K. Chaira and E. Marhrani, "Functional type Caristi-Kirk theorem on two metric spaces and applications," Fixed Point Theory and Applications, vol. 2016, no. 1, 2016.

[8] I. Ekeland, "On the variational principle," Journal of Mathematical Analysis and Applications, vol. 47, no. 2, pp. 324-353, 1974.

[9] I. Ekeland, "Sur les problemes variationnels," Comptes Rendus de l'Académie des Sciences, vol. 275, pp. 1057-1059, 1972.

[10] Z. Kadelburg, S. Radenovic, and S. Simic, "Metric spaces and Caristi-Nguyen-type theorems," Filomat, vol. 25, no. 3, pp. 111-124, 2011.

[11] E. Karapinar, F. Khojasteh, and W. Shatanawi, "Revisiting Cirić-type contraction with Caristi's approach," Symmetry, vol. 11, no. 6, p. 726, 2019.

[12] E. Karapinar, F. Khojasteh, and Z. D. Mitrovic, "A proposal for revisiting Banach and Caristi type theorems in b-metric spaces," Mathematics, vol. 7, no. 4, p. 308, 2019.

[13] W. A. Kirk and J. Caristi, "Mapping theorems in metric and Banach spaces," Bulletin of the Polish Academy of Sciences, vol. 25, pp. 891-894, 1975.

[14] M. R. Alfuraidan and M. A. Khamsi, "Caristi fixed point theorem in metric spaces with a graph," Abstract and Applied Analysis, vol. 2014, Article ID 303484, 5 pages, 2014.

[15] B. Banach, "Sur les opérations dans les ensembles abstraits et leur application aux équations intégrales," Fundamenta Mathematicae, vol. 3, pp. 133-181, 1992.

[16] L. B. Ciric, "A generalization of Banach's contraction principle," Proceedings of the American Mathematical Society, vol. 45, no. 2, pp. 267-273, 1974.

[17] L. B. Ćirić, "Generalized contractions and fixed point theorems," Publications de l'Institut Mathématique, vol. 12, pp. 19-26, 1971. 